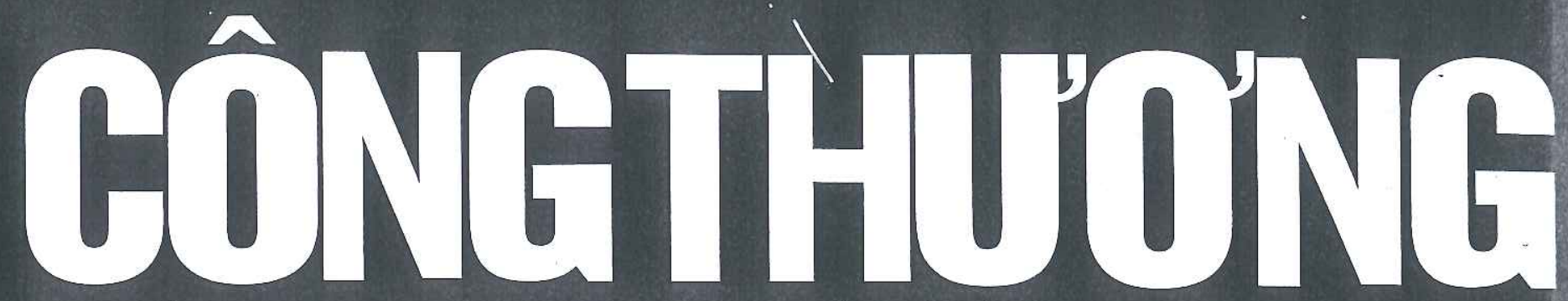

TAP CHÍ - CO QUAN THÔNG TIN LÝ LUÂN CỦA BỌ CÔNG THƯƠNG VIET NAM TRADE AND INDUSTRY REVIEW

\title{
CÁC KẾT QUẢ
}

NGHIÊN CUÚU KHOA HỌC

VÀ ỨNG DỤNG CÔNG NGHẸ 


\section{MỤC LỤC CONTENTS}

ISSN: 0866-7756 Số 12 - Tháng 11/2017

\section{LUẠT}

\section{NGUYẼ̃N THU HUONG}

Chính sách đâu tư theo hiệp định thương mại tự do Việt Nam - EU (EVFTA)

Investment policy under the Vietnam - EU free trade agreement (EVFTA).

\section{CHU THİ THÚY HẰNG}

Pháp luật Việt Nam với việc thể chế hóa quy định của Hiến pháp 2013 về quyền suy đoán vô tội

Vietnamese law with the intergration the constitution 2013 on the right to presumption of innocence..... 15

\section{LE THANH HOA - LE HUYNH PHUONG CHINH}

The patterns of inward fdi under the "DOI MOI" policy

Các yếu tố liên quan đến chính sách thu hút vốn đầu tư trực tiếp FDI trong thời kỳ "Đổi mới"

\section{NGUYẼNN THANH PHONG}

Đặc điểm tội phạm do người chưa thành niên thực hiện ở địa bàn các tỉnh Tây Nam bộ và những giải pháp phòng ngửa

Characteristics of juvenile delinquency in southwestern provinces and preventive measures

\section{TRƯONG VĂN DŨNG}

Bán hàng đa cấp và những vấn đề pháp lý đặt ra

Multi-level marketing and legal issues

\section{ĐặNG CôNG HIẾN}

Giải pháp nâng cao hiệu quả thực hiện pháp luật về an toàn thực phẩm trong hoạt động thương mại ở Việt Nam Solutions to improve the implementation of the law on food safety in commercial activities in Vietnam

\section{NGUYỄN HỮU KHÁNH LINH - NGUYẼ̃N THỊ HANH}

Các khía cạnh pháp lý về bảo hộ thông tin bí mật chống cạnh tranh không lành mạnh

theo quy định pháp luật quốc tễ

Legal elements for the protection of information concerning unfair competition

in compliance with international legislation 
NGÔ THİ LOAN - TRÂN HÂAU NGỌC - TRIINH MINH TÂM - NGUYỄN MAI PHƯƠNG - ĐÕ̃ SƠN TÙNG

Lý thuyễt về phương pháp định giá công nghệ TechFactor: Khuyễn nghị một số điều kiện áp dụng cho Việt Nam

The theory of Techfactor Method: Some recommended conditions for Vietnam.

\section{TRAN NGOC SON}

Business intelligence trends of enterprises in the time of industrial revolution 4.0

Kinh doanh thông minh - Xu thế của các doanh nighiệp trong thời đại cách mạng công nghiệp 4.0

\section{GIANG THANH LONG}

Một số biện pháp đổi mới công tác xuất nhập khẩu của các doanh nghiệp FDI

tại Hải Phòng theo hướng cuộc cách mạng 4.0

Some measures to improve the trading activities of FDI enterprises

in Hai Phong in the context of Industrial Revolution 4.0.

\section{QUẢN TRI - QUẢN LÝ}

\section{TRÂN VIẸTT HƯNG}

Kinh nghiệm quản trị chuỗi cung ứng sản phẩm cá ngừ Nhật Bản và mô hình áp dụng ở Việt Nam

Experience of supply chain management of tuna products in Japan and model applied in Vietnam

\section{HÀ NAM KHÁNH GIAO - VŨ THİ KIM XUÂN}

Các yẽu tỗ ảnh hưởng đễn lòng trung thành của nhân viên BIDV - Chi nhánh Bắc Sài Gòn

Factors affecting the loyalty of BIDV employees - Northern Branch of Saigon

\section{HÀ THI THU HÒA - TRẦ HOÀI NAM}

Phân tích các yếu tô ảnh hưởng đẽn quyễt định dừng canh tác khoai tây của nông hộ

tại xã Xuân Thọ, thành phố Đà Lạt, tỉnh Lâm Đồng

An analysis of factors effecting farmers' decision to stop producing potatoes

in Xuan Tho Commune, Dalat City, Lam Dong Province

\section{NGUYẼ̃N THI OANH}

Một số giải pháp đào tạo nguồn nhân lực chất lượng cao cho các doanh nghiệp Hàn Quốc đầu tư tại Việt Nam

High quality human resource training solutions for Korean enterprises investing in Vietnam.

\section{NGUYẼ̃N HOÀI NHÂN - VŨ THİNH TRƯờNG}

Đánh giá việc khai thác các giá trị văn hóa Chăm An Giang trong các chương trình du lịch tiêu biểu đễn tỉnh An Giang Assessing the exploitation of Cham An Giangcultural values in typical tourism programs in An Giang province.

\section{NGUYẼ̃N THI HOÀNG QUYÊN}

Đề xuất mô hình dự báo nhân lực Phú Quỗc giai đoạn 2016-2020

Proposing Phu Quoc human resource for casting model in the period 2016-2020. 


\title{
CÁC YẾU TỐ ẢNH HƯởNG ĐẾN LÒNG TRUNG THÀNH CỦA NHÂN VIÊN BIDV CHI NHÁNH BẮC SÀI GÒN
}

\author{
HÀ NAM KHÁNH GIAO - VŨ TH! KIM XUÂN
}

\section{TÓM TĂT:}

Nghiên cứu kiểm định mức độ tác động của các yếu tố ảnh hưởng đến lòng trung thành của nhân viên Ngân hàng Thương mại cổ phần Đầu tư và Phát triển Việt Nam - Chi nhánh Bắc Sài Gòn (BIDV- BSG), bằng việc khảo sát 208 nhân viên. Phương pháp phân tích Cronbachs Alpha, phân tích EFA cùng với phân tích hồi quy bội được sử dụng với phương tiện SPSS.

Kết quả cho thấy mức độ ảnh hưởng của các yếu tố đến đến lòng trung thành (TT) của nhân viên đối với BIDV- BSG - theo tầm quan trọng giảm dần: Thu nhập và phúc lợi, Cấp trên, Cơ hội đào tạo và phát triển, Hệ thống đánh giá thực hiện công việc, Mối quan hệ đồng nghiệp, Đặc điểm công việc. Nghiên cứu đề ra một số hàm ý quản trị cho các nhà quản lý BIDV- BSG nhằm nâng cao lòng trung thành của nhân viên.

Từ khóa: Lòng trung thành của nhân viên, Ngân hàng Thương mại cổ phần Đầu tư và Phát triển Việt Nam - Chi nhánh Bắc Sài Gòn, yếu tố ảnh hưởng.

\section{Giới thiệu}

Việc xây dựng đội ngũ nhân sự có chất lượng và gắn bó lâu dài với doanh nghiệp luôn là mối quan tâm được đặt lên hàng đầu. Một doanh nghiệp thành công và hoạt động hiệu quả bao giờ cũng nhờ đến sự đóng góp của đội ngũ nhân viên giỏi và trung thành. BIDV BSG trải qua 12 năm phát triển, đội ngũ nhân sự bản đầu chỉ 48 người, nay hơn 200 người. Tỷ lệ nhân viên làm việc trên 05 năm chiếm hơn $60 \%$, rất nhiều nhân viên đã gắn bó với chi nhánh kể từ ngày đầu thành lập. Trong những năm gần đây, trước những biến động chung của ngành Ngân hàng, BIDV'nói chung và $B I D V$ BSG nói riêng cũng đã gặp rất nhiều khó khăn và thách thức, đặc biệt là ở mảng nhân sự. Việc phân tích các yếu tố ảnh hưởng đến TT trở nên cần thiết.

2. Cơ sở lý thuyết và mô hình nghiên cứu

2.1. Lòng trung thành của nhân viên với tổ chức Lòng trung thành được hiểu rộng rãi như là một
' nhân viên có cảm giác gắn bó với tổ chức (Buchanan, 1974). Reichheld (1996) định nghĩa lòng trung thành là khi sã̃n sàng đầu tư hoặc hy sinh lợi ích cá nhân cho việc tăng cường một mối quan hệ nào đó. Trong môi trường kinh doanh ngày nay, các công ty muốn thành công đều phải phụ thuộc rất nhiều vào nỗ lực của các nhân viên giỏi của họ.

Theo Cooil và ctg (2007), các công ty có nhân viên trung thành nhiều hơn sẽ có nhiều lợi thế cạnh tranh hơn, tỷ lệ tồn tại cũng như thành công cao hơn so với các công ty có nhân viên trung thành ít hơn. Yee \& Faziharudean (2010) phân tích ảnh hưởng của lòng trung thành nhân viên đến hiệu suất của công ty, cho thấy nhân viên trung thành ảnh hưởng rất lớn đến công ty qua hai khía cạnh lợi nhuận thông qua chất lượng dịch vụ, sự thỏa mãn khách hàng và lòng trung thành khách hàng. Foster và ctg (2008) cũng chỉ ra rằng ở tại công ty 
mà có lòng trung thành lớn hơn giữa nhân viên, khách hàng và các cổ đông thì sẽ tạo ra được nhiều lợi nhuận hơn. Michlitsch (2000) đã kết luận các công ty có thể triển khai các chiến lược của họ một cách tốt nhất nếu họ đã phát triển và duy trì được đội ngũ nhân viên trung thành làm việc với hiệu suất cao dựa trên các yếu tố như: nhiệm vụ rõ ràng, chọn lọc và đào tạo, văn hóa doanh nghiệp, thông tin và truyền thông tốt và phần thưởng công

Turkyilmaz và ctg (2011) đánh giá các yếu tố tác động đến sự hài lòng và lòng trung thành của nhân viên trong tổ chức tại Thổ Nhĩ Kỳ chỉ ra 05 yếu tố: Cơ hội đào tạo và phát triển, Sự ghi nhận, Trao quyền, Làm việc nhóm, Điều kiện làm việc. Waqas và cộng sự (2014) đánh giá tác động trực tiếp của các yếu tố đến lòng trung thành của nhân viên trong công ty tại Pakistan, cho thấy 05 yếu tố: Tham gia vào quá trình ra quyết định, Được trao quyền, Phần thưởng và ghi nhận, Sự hài lòng, Điều kiện làm việc. Hassan và ctg (2013) đánh giá các yếu tố tác động đến sự hài lòng và lòng trung thành của nhân viên đang làm việc trong các ngân hàng vốn có vốn sở hữu nhà nước tại Pakistan, cho thấy 03 yếu tố: Lương và thưởng, Được trao quyền, Hệ thống đánh giá.

Tại Việt Nam, Trần Đình Mẫn Duy (2012) đánh giá các yếu tố tác động đến lòng trung thành của nhân viên tại Công ty cổ phần Đồng Tâm cho thấy 03 yếu tố: Bản chất công việc, Lãnh đạo, Lương thưởng. Mai Ngọc Khương và Bùi Diễ்m Tiên (2013) đánh giá các yếu tố tác động trực tiếp đến lòng trung thành của nhân viên và tác động gián tiếp đến lòng trung thành thông qua sự hài lòng cho thấy 03 yếu tố: Sự hỗ trợ của cấp trên, Làm việc nhóm, Điều kiện làm việc. Lê Trung Tín (2016) đánh giá các yếu tố tác động đến lòng trung thành của nhân viên tại Công ty Greenfield cho thấy 05 yếu tố: Lương, Khen thưởng, Đào tạo và phát triển, Đồng nghiệp, Cấp trên.

\subsection{Mô hình nghiên cúu đề xuất}

Từ cơ sở các lý thuyết và các nghiên cứu liên quan, cũng như kết quả nghiên cứu định tính, mô hình đề xuất gồm 06 yếu tố: $\mathrm{TT}=\mathrm{f}$ (Thu nhập và phúc lợi, Cơ hội đào tạo và thăng tiến, Cấp trên, Đặc điểm công việc, Môi trường làm việc, Trao quyền, Mối quan hệ với đồng nghiệp, Hệ thống đánh giá thực hiện công việc) và các giả thuyết nghiên cứu.

$H_{1}$ : Thu nhập và phúc lợi ảnh hưởng thuận ch đến TT.

$\mathrm{H}_{2}$ : Cơ hội đào tạo và thăng tiến ảnh hu thuận chiều đến TT.

$\mathrm{H}_{3}$ : Cấp trên ảnh hưởng thuận chiều đến TT

$H_{4}$ : Đặc điểm công việc ảnh hưởng thuận ch đến TT.

$H_{5}$ : Môi trường làm việc ảnh hưởng thuận ch đến TT.

$H_{6}$ : Trao quyền ảnh hưởng thuận chiều đến

$H_{7}$ : Mối quan hệ với đồng nghiệp ảnh hư thuận chiều đến TT.

$H_{8}$ : Hệ thống đánh giá thực hiện công việc a hưởng thuận chiều đến TT.

\section{Kết quả nghiên cứu}

\subsection{Mô tả dũ liệu}

Khảo sát trực tiếp toàn bộ 209 nhân viên đa làm việc tại $\mathrm{BIDV} B S G$, thu về 209 bảng, loại 01 phiếu không đạt yêu cầu, còn lại là 208 bả

Bảng 1. Bảng thống kê mô tă

\begin{tabular}{|l|c|c|}
\hline & Tần số & Tỷ lệ \% \\
\hline Giới tính & 208 & 100,0 \\
\hline Nữ & 102 & 49,0 \\
\hline Nam & 106 & 51,0 \\
\hline Nhóm tuổi & 208 & 100,0 \\
\hline Tù 18-25 & 13 & 6,3 \\
\hline Tù̀ 26-35 & 52 & 25,0 \\
\hline Tù̀ 36-45 & 74 & 35,6 \\
\hline Trên 45 & 69 & 33,2 \\
\hline Trình độ & 208 & 100,0 \\
\hline Tù̀ Trung cấp trở xuống & 14 & 6,7 \\
\hline Cao đăhng & 65 & 31,3 \\
\hline Đại học & 123 & 59,1 \\
\hline Sau đại học & 6 & 2,9 \\
\hline Thu nhập & 208 & 100,0 \\
\hline$<5$ triệu & 4 & 1,9 \\
\hline 5 - 10 triệu & 70 & 33,7 \\
\hline$>10$ - 15 triệu & 105 & 50,5 \\
\hline$>15$ triệu & 29 & 13,9 \\
\hline & & \\
\hline
\end{tabular}

(Nguồn: Xủ lý tù dũ liệu khảo sát) 
Bảng 2. Kết quả Cronbachs Alpha

\begin{tabular}{|l|c|c|c|c|}
\hline \multicolumn{1}{|c|}{ Thang đo } & Ký hiệu & $\begin{array}{c}\text { Số biến } \\
\text { quan sát }\end{array}$ & $\begin{array}{c}\text { Hệ số tương quan } \\
\text { biến- tổng nhỏ nhất }\end{array}$ & $\begin{array}{c}\text { Cronbach's } \\
\text { Alpha }\end{array}$ \\
\hline Thu nhập và phúc lọi & TP & 5 & 0,711 & 0,899 \\
\hline Cơ hội đào tạo và phát triển & ĐP & 5 & 0,549 & 0,834 \\
\hline Cấp trên & CT & 5 & 0,587 & 0,841 \\
\hline Gặc điểm công việc & CV & 4 & 0,556 & 0,766 \\
\hline Môi trường làm việc & MT & 4 & 0,746 & 0,893 \\
\hline Trao quyê̂n & TQ & 3 & 0,698 & 0,849 \\
\hline Mối quan hệ đồng nghiệp & ĐN & 4 & 0,551 & 0,776 \\
\hline Hệ thống đánh giá thụ̣c hiện công việc & DG & 4 & 0,540 & 0,776 \\
\hline Lòng trung thành của nhân viên & TT & 4 & 0,542 & 0,821 \\
\hline
\end{tabular}

(Nguồn: Xủ lý tù dũ liệu khảo sát)

(tỷ lệ 99,52\%).

\section{2. Đánh giá thang đo bằng hệ số tin cậy Cronbachs Alpha}

Sau khi loại bỏ biến CV1, kiểm định Cronbachs Alpha cho kết quả đều lớn hơn 0,6 , hệ số tương quan biến tổng đều lớn hơn 0,3 (Bảng 2), cho thấy các thang đo đều đạt được độ tin cậy, 38 biến quan sát được giữ lại.

\subsection{Phân tích nhân tố khám phá EFA}

34 biến độc lập được đưa vào phân tích yếu tố EFA với phương pháp trích Principal Component và phép quay Varimax. Kết quả kiểm định Barlett với sig bằng $0(<0,005)$ và hệ số KMO bằng $0,732(>$ $0,5)$, phương sai trích là $67,159 \%(>50 \%)$ đạt yêu cầu. Mô hình hồi quy có 08 biến độc lập. (Bảng 3).

Kết quả phân tích EFA TT với phương pháp Principal Component Analysis, phép xoay Varimax, trích được 01 yếu tố với 4 biến quan sát và phương sai trích tích lũy $65,259 \%$ (> 50\%), Giá trị Eigenvalue là 2,610 (>1), KMO là 0,756 thỏa mãn yêu cầu, kiểm định Bartletts có ý nghĩa thống kê $(\operatorname{Sig}=0,000)$ nên các biến quan sát có tương quan với nhau trong tổng thể.

\subsection{Phân tích tương quan}

Ma trận tương quan Pearson cho thấy mức ý nghĩa của các hệ số rất nhỏ $(\operatorname{sig}<0,10)$ nên các hệ số tương quan có ý nghĩa thống kê và đủ điều kiện để đưa vào phân tích hồi quy. Cần quan tâm các yếu tố $\mathrm{CV}, \mathrm{MT}$, ĐN do sig > 0,05.

\subsection{Phân tích hồi quy}

Kết quả hồi quy sau khi loại biến Trao quyền (TQ) và Môi trường làm việc (MT) do không có ý nghĩa thống kê $(\operatorname{Sig}=0,230$ và $\operatorname{Sig}=0,265$ ) như

\section{Bảng 4. Kết quả hồi quy}

\begin{tabular}{|c|c|c|c|c|c|}
\hline \multirow{2}{*}{$\begin{array}{l}\text { Biến } \\
\text { độc lập }\end{array}$} & \multirow{2}{*}{$\begin{array}{c}\text { Hê số } \\
\text { hồi quy } \\
\text { (B) }\end{array}$} & \multirow{2}{*}{$\begin{array}{l}\text { Hệ số } \\
\text { Beta }\end{array}$} & \multirow{2}{*}{$\begin{array}{l}\text { Mứcý } \\
\text { nghĩa }\end{array}$} & \multicolumn{2}{|c|}{$\begin{array}{l}\text { Thống kê } \\
\text { đa cộng tuyến }\end{array}$} \\
\hline & & & & $\begin{array}{c}\text { Dung } \\
\text { sai }\end{array}$ & VIF \\
\hline (Hằng số) & $-0,539$ & 0,276 & & 0,052 & \\
\hline TP & 0,354 & 0,065 & 0,341 & 0,000 & 0,545 \\
\hline$\boxminus P$ & 0,196 & 0,071 & 0,177 & 0,006 & 0,515 \\
\hline CT & 0,326 & 0,067 & 0,299 & 0,000 & 0,565 \\
\hline CV & 0,083 & 0,039 & 0,103 & 0,032 & 0,928 \\
\hline EN & 0,072 & 0,033 & 0,108 & 0,029 & 0,882 \\
\hline$Đ G$ & 0,144 & 0,047 & 0,146 & 0,003 & 0,932 \\
\hline
\end{tabular}

$R^{2}$ hiệu chỉnh: 0,563

Thống kê F (ANOVA): 45,409

Múc ý nghĩa (Sig. của ANOVA): 0,000

Durbin-Watson: 1,973

(Nguồn: Xủ lý tù dũ liệu khảo sát)

trong Bảng 4.

Kết quả phân tích phượng sai ANOVA cho thấy trị thống kê $\mathrm{F}=45,409$ với giá trị sig $=0,000$ chứng tỏ mô hình hồi quy phù hợp với tập dữ liệu. Durbin-Watson là $1,973<2$ cho thấy không có sự tương quan giữa các biến trong mô hình. Hệ số VIF của các biến đều $<10$ chứng tỏ không xảy ra hiện tượng đa cộng tuyến. Hệ số R2 hiệu chỉnh 0,563 cho thấy $56,3 \%$ sự biến thiên của biến phụ thuộc được giải thích bởi các biến độc lập. Phương trình hồi quy chưa chuẩn hóa: $\mathrm{TT}=-0,539+0,354 * \mathrm{TP}$ $+0,196 * \mathrm{DP}+0,326 * \mathrm{CT}+0,083 * \mathrm{CV}+0,072 * \mathrm{DN}$ $+0,144 * \mathrm{BG}$.

3.6. Kiểm định sụ vi phạm của các giả địinh hồi qui 


\section{TẠP CHÍ CÔNG THỬัNG}

Bảng 3. Kết quå EFA

\begin{tabular}{|c|c|c|c|c|c|c|c|c|}
\hline \multirow{2}{*}{ Biến quan sát } & \multicolumn{8}{|c|}{ Hệ số tải nhân tố } \\
\hline & 1 & 2 & 3 & 4 & 5 & 6 & 7 & 8 \\
\hline $\mathrm{TP}_{3}$ & 0,865 & & & & & & & \\
\hline $\mathrm{TP}_{1}$ & 0,857 & & & & & & & \\
\hline $\mathrm{TP}_{4}$ & 0,839 & & & & & & & \\
\hline $\mathrm{TP}_{2}$ & 0,824 & & & & & $\therefore$ & & \\
\hline TP5 & 0,815 & & & & & & & \\
\hline $\mathrm{MT}_{1}$ & & 0,880 & & & & & & \\
\hline $\mathrm{MT}_{2}$ & & 0,862 & & & & & & \\
\hline $\mathrm{MT}_{4}$ & & 0,860 & & & & & & \\
\hline $\mathrm{MT}_{3}$ & & 0,860 & & & & & & \\
\hline $\mathrm{CT}_{2}$ & & & 0,829 & & & & & \\
\hline $\mathrm{CT}_{4}$ & & & 0,816 & & & & & \\
\hline $\mathrm{CT}_{3}$ & & & 0,807 & & & & & \\
\hline $\mathrm{CT}_{1}$ & & & 0,728 & & & & & \\
\hline $\mathrm{CT}_{5}$ & & & 0,715 & & & & & \\
\hline $\mathrm{DP}_{4}$ & & & & 0,831 & & & & \\
\hline $\mathrm{BP}_{3}$ & & & & 0,826 & . & & & \\
\hline $\mathrm{DP}_{1}$ & & & & 0,788 & & & & \\
\hline $\mathrm{EP}_{5}$ & & & & 0,714 & & & & \\
\hline $\mathrm{EP}_{2}$ & & & & 0,707 & 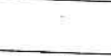 & & & \\
\hline$\boxminus G_{3}$ & & & & & 0,795 & & & \\
\hline $\mathrm{DG}_{2}$ & & & & & 0,789 & & & \\
\hline$Đ G_{4}$ & & & & . & 0,760 & & & \\
\hline$Đ G_{1}$ & & & & & 0,748 & & & \\
\hline$\boxminus \mathrm{N}_{1}$ & & & ' & .. & & 0,802 & & \\
\hline $\mathrm{BN}_{4}$ & & & & & & 0,763 & . & \\
\hline $\mathrm{BN}_{3}$ & & & & & & 0,756 & & \\
\hline $\mathrm{BN}_{2}$ & & & & & & 0,755 & & \\
\hline $\mathrm{CV}_{3}$ & & & & & & & 0,794 & \\
\hline $\mathrm{CV}_{4}$ & & & & & & & 0,761 & \\
\hline $\mathrm{CV}_{5}$ & & & & & & & 0,753 & \\
\hline $\mathrm{CV}_{2}$ & & & & . & & & 0,752 & \\
\hline $\mathrm{TQ}_{3}$ & & & & & & & & 0,890 \\
\hline$T Q_{1}$ & & & & & & & & 0,866 \\
\hline $\mathrm{TQ}_{2}$ & & & & & & & & 0,846 \\
\hline Eigenvalue & 3,875 & 3,628 & 3,107 & 3,018 & 2,668 & 2,447 & 2,273 & 1,817 \\
\hline Phương sai trích \% & 11,398 & 10,67 & 9,137 & 8,877 & 7,847 & 7,198 & 6,687 & 5,345 \\
\hline Phương sai tích lũy & 11,398 & 22,068 & 31,206 & 40,083 & 47,93 & 55,128 & 61,814 & 67,159 \\
\hline
\end{tabular}

(Nguồn: Xủ̉ lý tù dũ liệu khảo sát) 
Đồ thị phân tán Scatterplot cho thấy phần dư phân tán ngẫu nhiên xung quanh đường đi qua tung độ 0 và dao động nhiều ở biên độ $+/-1$, chứng tỏ rằng giả định liên hệ tuyến tính không bị vi phạm. Biểu đồ tần số Histogram cho thấy đường cong phân phối chuẩn đặt chồng lên biểu đồ tần số, giá trị trung bình rất nhỏ gần bằng 0 (mean $=-4,12 \mathrm{E}$ $15)$ và độ lệch chuẩn gần bằng 1 (Std. $\mathrm{Dev}=$ $0,985)$, cho thấy phân phối của phần dư xấp xỉ chuẩn. Biểu đồ P-P plot cũng cho thấy các điểm quan sát không phân tán quá xa đường thẳng kỳ vọng nên có thể kết luận rằng giả thiết phần dư có phân phối chuẩn không bị vi phạm. Như vậy, mô hình hồi qui tuyến tính trên là phù hợp.

Bảng 4 cho thấy 06 biến độc lập có tác động cùng chiều vào TT theo thứ tự giảm dần: Thu nhập và phúc lợi $\left(\beta_{1}=0,341\right)$, Cấp trên $\left(\beta_{3}=0,299\right)$, Cơ hội đào tạo và phát triển $\left(\beta_{2}=0,177\right)$, Hệ thống đánh giá thực hiện công việc $\left(\beta_{8}=0,146\right)$, Mối quan hệ đồng nghiệp $\left(\beta_{7}=0,108\right)$, Đặc điểm công việc $\left(\beta_{4}=0,103\right)$. Các giả thuyết $\mathrm{H}_{1}, \mathrm{H}_{2}, \mathrm{H}_{3}, \mathrm{H}_{4}$, $\mathrm{H}_{7}, \mathrm{H}_{8}$ được chấp nhận ở độ tin cậy $95 \%$, bác bỏ giả thuyết $\mathrm{H}_{5}$ và $\mathrm{H}_{6}$.

\subsection{Kiểm định sư khác biệt}

Kiểm định $\mathrm{t}$-test cho giới tính và kiểm định ANOVA một chiều cho nhóm tuổi, thu nhập và trình độ. Kết quả cho thấy không có sự khác biệt giữa các giới tính, nhóm tuổi, thu nhập và trình độ về TT.

\section{Kết luận và đề xuất hàm ý quản trị \\ 4.1. Kết luân}

Qua quá trình nghiên cứu, nhóm tác giả đã sử dụng phương pháp nghiên cứu định lượng phù hợp, xử lý số liệu bằng phương tiện thống kê để có thể xác định được 06 yếu tố ảnh hưởng đến $\mathrm{TT}$, sắp xếp theo thứ tự tác động giảm dần: Thu nhập và phúc lợi, Cấp trên, Cơ hội đào tạo và phát triển, Hệ thống đánh giá thực hiện công việc, Mối quan hệ đồng nghiệp, Đặc điểm công việc. Nghiên cứu chưa chỉ ra được có sự khác biệt về TT theo giới tính, độ tuổi, trình độ, thu nhập ở độ tin cậy 95\%.

\subsection{Hàm ý quản trị}

- Thu nhập và phúc lợi

Đây là yếu tố ảnh hưởng lớn nhất với mean = 3,293 . Bên canh việc tiếp tục duy trì các phúc lợi cơ bản, BIDV BSG cần lưu ý các chính sách phúc lợi cộng thêm cho nhân viên: hỗ trợ cho nhân viên vay mua nhà ở, vay vốn ưu đãi về lãi suất... Cần có những chương trình phúc lợi đa dạng như bình chọn, khen thưởng nhân viên hàng tháng. BIDV BSG cần điều chỉnh một số chính sách lương, thưởng, hoa hồng... phù hợp với kết quả đóng góp. BIDV BSG cần tham khảo, so sánh thu nhập tại các ngân hàng khác cùng ngành, để có mức thu nhập phù hợp cho từng người lao động. BIDV BSG cần có bảng mô tả công việc cho từng vị trí để người lao động thấy được vai trò và đóng góp của mình.

\section{- Cấp trên}

Mean của nhóm yếu tố này là 2,817 , khá thấp. Lãnh đạo cần có sự đánh giá khách quan, công bằng, minh bạch với nhân viên, theo các tiêu chí rõ ràng, tránh hiểu nhầm, tránh suy diễn. Lãnh đạo cần không ngừng học hỏi nâng cao cả năng lực lãnh đạo và kiến thức chuyên môn. Cần tổ chức các buổi gặp mặt nói chuyện thân mật giữa lãnh đạo cấp cao và nhân viên để xây dựng mối quan hệ này tốt hơn. Cấp trên cũng cần phải ghi nhận đóng góp của người lao động khi họ đạt được các mục tiêu. Cấp trên cần hiểu rõ hơn về người lao động, cần trao đổi, tìm hiểu về sở thích, điểm mạnh, điểm yếu của người lao động trong công việc cũng như trong cuộc sống.

\section{- Cơ hội đào tạo và phát triển}

Mean của nhóm yếu tố này là 3,317. Mỗi phòng ban, bộ phận nên xây dựng quy trình xử lý các công việc từ đơn giản đến phức tạp; tài liệu này dùng để tham chiếu, hướng dẫn cho nhân viên, các quy trình này nên thường xuyên được cập nhật. BIDV BSG cần xây dựng lộ trình phát triển nighề nghiệp cụ thể cho từng vị trí công việc. BIDV BSG cần quan tâm hơn nữa trong đào tạo kỹ năng cho người lao động: nói chuyện qua điện thoại, quản lý thời gian, đàm phán... BIDV BSG cần xây dựng chế độ thăng tiến đặc biệt cho những người lao động giỏi, những người lao động có thành tích xuất sắc. BIDV BSG cần lập ra chương trình đào tạo nội bộ để nâng cao hiệu quả đào tạo.

\section{- Hệ thống đánh giá thực hiện công việc}

Mean của nhóm yếu tố này là 3,490 . BIDV BSG nên xây dựng các tiêu chí đánh giá riêng cho từng chức danh công việc, từng bộ phận, phòng ban để việc đánh giá được chính xác, tránh cảm tính. $\mathrm{BIDV}$ BSG nên xây dựng hệ thống đo lường công việc được lượng hóa bằng các chỉ tiêu rõ ràng, khách quan, việc khen thưởng, kỷ luật cũng nên áp dụng 
các quy chế đó một cách công khai, minh bạch BIDV BSG cần có chính sách thăng tiến minh bạch, rõ ràng dựa vào kết quả đánh giá công việc.

- Mối quan hệ với đồng nghiệp

Mean của nhóm yếu tố này là 3,409 . BIDV BSG nên tổ chức những buổi gặp gỡ, trao đổi kinh nghiệm, giao lưu chia sẻ công việc giữa các cấp lãnh đạo, giữa các phòng ban. Trong cùng phòng ban, nên có sự hỗ trợ công việc giữa các đồng nghiệp, tránh quá tải. BIDV BSG nên thực hiện thường xuyên hơn các buổi giao lưu giữa các phòng ban. Công đoàn, Đoàn thanh niên tổ chức các buổi dã ngoại, team building cho nhân viên để nâng cao tính đoàn kết. BIDV BSG nên thường xuyên tổ chức các cuộc thi nghiệp vụ giữa các phòng/tổ, để nhân viên tự rà soát lại kiến thức, kỹ năng, tăng cường tính đoàn kết. Những chính sách đãi ngộ của BIDV BSG, kể cả hưu trí, cũng là yếu tố giúp cho nhân viên tin tưởng và gắn bó.

- Đặc điểm công việc

Mean của nhóm yếu tố này là 3,644 . Ngân hàng nên tập trung nhiều hơn và chi tiết hơn vào bảng mô tả công việc. BIDV BSG nên đào tạo nghiệp vụ, giúp nhân viên hiểu biết đúng đắn về vị trí, trách nhiệm, tính tương tác trong công việc. Sự gián đoạn hay chồng chéo, thiếu kết nối giữa các bộ phận cần được ngân hàng rà soát kỹ càng khi phân công công việc. BIDV BSG cũng nên xét tình hình thực tế, nguyện vọng và năng lực của nhân viên, tính chất các phòng ban để bố trí phù hợp. BIDV BSG nên thực hiện việc chọn đúng người ngay từ giai đoạn tuyển dụng nhân viên.

- Hạn chế của nghiên cứu và hướng nghiên cứu tiếp theo

Mặc dù đã giải quyết xong mục tiêu nghiên cứu đã đề ra, nhưng vẫn còn một số hạn chế: (1) Phương pháp lấy mẫu toàn bộ tại BIDV BSG, do đó kết quả nghiên cứu chỉ có ý nghĩa cho chi nhánh, (2) Nghiên cứu chỉ xem xét tác động nói chung của toàn bộ mẫu thu thập, chưa thực hiện phân tích sự khác biệt giữa các nhóm nhân viên: nhân viên tín dụng, giao dịch viên, nội bộ, kho quỹ... Đó cũng chính là gợi ý cho những nghiên cứu tiếp theo

\section{TÀI LIỆU THAM KHẢO:}

1. Buchanan, B. (1974), "Building Organizational Commitment: The Socialization of Managers in Work Organization”, Administrative Science Quarterly, 19, pp. 533-546.

2. Cooil, B., Timothy L. Keiningham, Lerzan Aksoy, and Michael Hsu (2007), "A Longitudinal Analysis of Customer Satisfaction and Share of Wallet: Investigating the Moderating Effect of Customer Characteristics",
Journal of Marketing, 71, pp. 67-83.

3. Foster, C., Whysall, P., \& Harris, L. (2008), "Employee loyalty: An exploration of staff commitment levels towards retailing, the retailer and the store", International Review of Retail, Distribution and
Consumer Research, 18, pp. $423-435$.

4. Hassan, et al (2013), Impact of HR Practices on Employee Satisfaction and Employee Loyalty: An Empirical Study of Government Owned Public Sector Banks of Pakistan, Middle-East Journal of Scientific
Research, 16, pp. 1- 8 . 5. Lê Trung Tín (2016), Các yếu tố tác động đến lòng trung thành của nhân viên tại công ty
GREENFIELD, Luận văn Thạc sĩ kinh tế, Trường Đại học Kinh tế Thành phố Hồ Chí Minh, TP. Hồ Chí
Minh.

6. Mai Ngoc Khuong và Bui Diem Tien (2013), "Factors influencing employee loyalty directly and of current research, 4, pp. 81 - 95 . 7. Michlitsch, J. F (2000), "High-performing, loyal employees: The real way to implement strategy",
Strategy \& Leadership, 28, pp. 28 - 33 .

8. Reichheld, F. F. (1996), The Loyalty Effect: The Hidden ForceBehind Growth, Profits, and Lasting

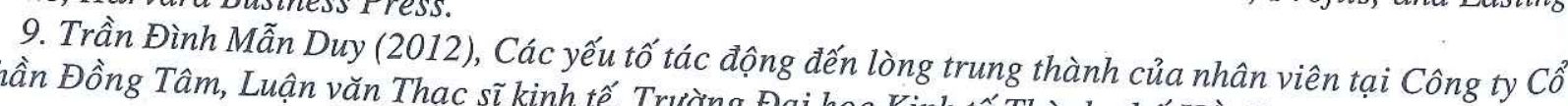
phần Đồng Tâm, Luận văn Thạc sĩ kinh tế, Trường Đại học Kinh tế Thành phố Hồ Chí Minh. 
10. Turkyilmaz, A., Akman, G., Ozkan, C., Pastuszak, Z. (2011), Empirical study of public sector employee loyalty and satisfaction, Industrial Management \& Data Systems; 111, pp. 675 - 696.

11. Waqas, et al (2014), "Factors Influencing Job Satisfaction and Its Impact on Job Loyalty", International Journal of Learning \& Development, 4, pp. 141 - 161.

12. Yee, B. Y., \& Faziharudean, T. M. (2010), Factors Affecting Customer Loyalty of Using Internet Banking in Malaysia, Journal of Electronic Banking Systems, 2 1, pp. 31- 57.

Ngày nhận bài: 3/9/2017

Ngày phản biện đánh giá và sửa chữa: 13/9/2017

Ngày chấp nhận đăng bài: 23/9/2017

Thông tin tác giả:

\title{
1. HÀ NAM KHÁNH GIAO
}

Trưởng khoa Đào tạo Sau đại học, Trường Đại học Tài chính - Marketing

Email: khanhgiaohn@yahoo.com

Điện thoại: 0903306363

\section{VŨ THI KIM XUÂN}

Phó Giám đốc NH TMCP Đầu tư và Phát triển Việt Namm - Chi nhánh Bắc Sài Gòn

Email: vtkimxuan@gmail.com

Điện thoại: 0982070274

\section{FACTORS AFFECTING THE LOYALTY OF BIDV EMPLOYEES - NORTHERN BRANCH OF SAIGON}

\author{
- HA NAM KHANH GIAO \\ Post Graduate Deparment - University of Finance and Marketing \\ - VU THI KIM XUAN \\ Vice Director of Bank for Investment and Development of Vietnam \\ Norhtern Sai Gon Bra nch
}

\section{ABSTRACT:}

The research investigates how the factors affecting staff loyalty on Bank of Investment and Development Vietnam - Northern Saigon branch (BIDV- NSB), by intervewing 208 employees. The method of Cronbachs Alpha analysis, EFA analysis and multiple regression analysis were used with the SPSS program.

The result shows that the affects of the factors on custom ers loyalty on BIDV- NSB are: Income and Bonus, Supervisor relationship, Opportunities for training and development, Evaluation system of work achievement, Peer relationship, Work characteris tics. The research also suggests some solutions to branch management to enhance the staff loyalty .

Keywords: Staff loyalty, Bank of Investment and Deve1 opment Vietnam- North Saigon branch, affecting factors. 